\title{
Separation, Gharacteristics and Minimal Amino-Acid Requirements of Six Variants Derived from a Strain of Bacillus cereus
}

\author{
BY W. B. MOORE \\ The Wellcome Research Laboratories (Biological Division), \\ Langley Court, Beckenham, Kent
}

(Received 16 February 1965)

\section{SUMMARY}

Maintenance of a stock culture of Bacillus cereus by subculture on nutrient agar plates resulted in dissociation of the culture manifest as the development of fimbriate outgrowths from the initially entire peripheries of confluent streaks and isolated colonies. Auxanographic determination of minimal amino acid requirements of the dissociated culture indicated that it was composed of at least two types of organism: one was sensitive to (i.e. probably lysed by) L-lysine and the other resistant. They were further separated on the basis of sensitivity to lysine and colonial morphology into six different types. The microscopic appearance of organisms and their arrangement within colonies indicated that the original culture and each of the variants was a rough $(R)$ type. At $28^{\circ}$, four variants required only L-glutamate for growth, while the remaining two required L-cysteine or $L$ - $\alpha$-alanine in addition. At $35^{\circ}$ a greater number of amino acids had to be supplied for growth to occur. At neither temperature did the variants show a requirement for growth factors of the vitamin B-complex. These nutritional requirements, together with infrared spectra of whole organisms and biochemical characteristics determined by conventional methods, confirmed that each variant, though showing minor differences, did represent a culture of $B$. cereus. On auxanographic plates in the presence of L-lysine at $28^{\circ}$, three variants were lysed, one was unaffected, and the growth of two enhanced.

\section{INTRODUCTION}

The ease with which bacteria, and in particular the aerobic sporeformers, dissociate is well known and is one cause of the difficulties encountered in identification and classification of members of the genus Bacillus. Though there have been many studies of dissociation of Bacillus (Smith, Gordon \& Clark, 1952; Braun, 1947), few have been concerned with detecting alterations in metabolic and nutritional patterns. The dissociation has usually been correlated with change of colonial morphology or capacity to form a particular metabolic product. The requirements for growth factors and amino acids have been separately reported in some detail for a few members of the genus, namely: B. polymyxa and $\boldsymbol{B}$. macerans (Katznelson, 1944), B. larvae (Katznelson \& Lochhead, 1948), B. larvae White (Lochhead, 1942), B. alvei and B. para-alvei (Katznelson \& Lochhead, 1947), B. anthracis (Brewer et al. 1946), B. popilliae Dutky and B. lentimorbus Dutky (Dutky, 1947), B. subtilis (Teas 1950; Demain, 1958), and B. coagulans (Cleverdon, Pelczar \& Doetsch, 1949a, $b$; 
Campbell \& Sniff, 1959). The B vitamin and amino acid requirements of almost al of the species within the genus were reported by the comprehensive nutritional surveys of Knight \& Proom (1950) and Proom \& Knight (1955). Although this latter work provided data for a nutritional classification the elucidation of minimal amino acid requirements was not then necessary. In the present work the dissociation, cultural characteristics and minimal amino acid requirements of one species, $B$. cereus, have been investigated in greater detail.

\section{METHODS}

Amino acids. These were obtained from British Drug Houses Ltd., or Light and Co. Ltd. Before use each was checked for purity by paper chromatography.

Sugars. Glucose was of A.R. quality. The maltose sample used was found to contain appreciable quantities of several amino acids upon examination in the Beckman/Spinco Amino Acid Analyser; it is subsequently referred to as impure maltose.

Inorganic salts. All were of A.R. quality.

Water. Glass-distilled water, passed through an Elgastat de-ionizer to yield an effluent having 2 $-4 \mathrm{M} \Omega$ resistance, was used for making all solutions.

Culture flasks. The specially designed flasks (Moore, 1963) were made from soda glass having the following composition $(\%, w / w): \mathrm{SiO}_{2}, 71 \cdot 5 ; \mathrm{Al}_{2} \mathrm{O}_{3}, 2 \cdot 2 ; \mathrm{CaO}, 5 \cdot 7$; $\mathrm{MgO}, 3.0 ; \mathrm{BaO}, 1 \cdot 7 ; \mathrm{Na}_{2} \mathrm{O}, 14.0 ; \mathrm{K}_{2} \mathrm{O}, 1.5$. This analysis is given since, under the same growth conditions, equivalent flasks of Pyrex glass caused a marked lag in growth response.

Agar. The material used was of Korean origin.

Organism. Bacillus cereus strain CN 753 was selected from the Wellcome Research Laboratories Culture Collection.

Media. Nutrient broth was used for growing crops of organisms to be used subsequently as inocula. For nutritional investigations a chemically defined medium similar to that of Knight \& Proom (1950) was used. The complete basal medium (BM) contained (g./l. final volume): glucose (or maltose) $5.0 ; \mathbf{K H}_{2} \mathrm{PO}_{4}, 1.5 ;\left(\mathrm{NH}_{4}\right)_{2}$ $\mathrm{HPO}_{4}, 7 \cdot 0 ; \mathrm{MgSO}_{4} .7 \mathrm{H}_{2} \mathrm{O}, 0.5$; in $\mathrm{mg}$; $\mathrm{MnCl}_{2} .4 \mathrm{H}_{2} \mathrm{O}, 37 ; \mathrm{FeSO}_{4} .7 \mathrm{H}_{2} \mathrm{O}, 2.5$; $\left(\mathrm{NH}_{4}\right)_{6} \mathrm{Mo}_{7} \mathrm{O}_{24} \cdot 4 \mathrm{H}_{2} \mathrm{O}, 2 \cdot 0$.

Maintenance of cultures. (a) During the early phase of the work a freeze-dried stock culture was reconstituted in $\mathbf{0 . 5} \mathrm{ml}$. nutrient broth, incubated for $2-3 \mathrm{hr}$ at $28^{\circ}$ and then plated on nutrient agar to check purity. The culture was kept at room temperature and further maintained by fortnightly or monthly subculture on to plates of fresh medium. (b) Following the change in growth characteristics and subsequent separation of variants, freeze-dried stocks of each were stored at $4^{\circ}$.

Preparation of inocula for growth experiments. For each experiment the appropriate freeze-dried culture was reconstituted and plated to check purity and colonial morphology. A liquid culture in broth inoculated from a single typical colony was then grown at $28^{\circ}$ overnight. The organisms contained in a $6 \mathrm{ml}$. portion were then washed successively in three $6 \mathrm{ml}$. portions of sterile saline and finally suspended in a fourth $6 \mathrm{ml}$. portion. After determining the concentration of the suspension it was diluted to correspond to $85 \mu \mathrm{g}$. dry weight bacteria/ml. This suspension served as the inoculum, $0.4 \mathrm{ml}$. being added to about $20 \mathrm{ml}$. of final medium. Finally the suspension was plated to check viability, purity and colonial morphology. 
Inocula for auxanography. Crops of organisms were grown and washed as described above and enough bacterial suspension added to cooled molten agar medium to produce a concentration of organisms in the final plates corresponding to $10 \mu \mathrm{g}$. dry wt. bacteria/ml.

Measurement of amount of organism in inocula. The extinction of a known dilution of a washed cell suspension of organism was measured in $1 \mathrm{~cm}$. optical cells at $404 \mathrm{~m} \mu,\left(E_{404}\right)$ by using a Unicam SP 1400 spectrophotometer. The amount of organism, in terms of $\mu \mathrm{g}$. dry wt. bacteria $/ \mathrm{ml}$. suspension was then read from a calibration curve, and the value calculated for the undiluted suspension. The latter was then diluted before use to a concentration of organism equivalent to $85 \mu \mathrm{g}$. dry wt. bacteria/ml.

Measurement of growth. Extinctions of cultures in liquid chemically defined medium were read in situ by using specially designed culture flasks (Moore, 1963), the side-arms of which fitted into the optical cell carrier of the same spectrophotometer. The values are recorded in terms of $\mu \mathrm{g}$. dry wt. bacteria/ml.

Preparation of chemically defined medium. Each of the inorganic salts was kept as a concentrated aqueous stock solution at room temperature. Glucose, or maltose, solution $(25 \%, \mathrm{w} / \mathrm{v})$ in de-ionized water and separate solutions of amino acids in 0.25 M-phosphate buffer ( $\mathrm{pH} \mathrm{7.0)}$ ) were freshly made for each experiment.

Complete basal (BM) medium. Appropriate volumes of stock inorganic salt solutions were added to about $800 \mathrm{ml}$. de-ionized water, the mixture adjusted to $\mathrm{pH} 7 \cdot 5$ and the volume made to $900 \mathrm{ml}$. The mixture was then heated to boiling, cooled quickly to about $15^{\circ}$ and filtered (Whatman no. 1). The volume of the filtrate was readjusted to $900 \mathrm{ml}$. and to $\mathrm{pH} 7 \cdot 5$. Portions of $18 \mathrm{ml}$. were then distributed into culture vessels and sterilized. Glucose or maltose solution $(0.4 \mathrm{ml}$.) was added aseptically and the completed medium left for 18-24 $\mathrm{hr}$ at room temperature before being inoculated.

For auxanography, agar (2 g.) and BM medium (75 ml., sugar omitted) were autoclaved in admixture, and glucose or maltose solution $(4 \mathrm{ml}$.) added aseptically to the cooled mixture.

Complete chemically defined (CDM) medium. Solutions of amino acids were added asceptically to each of the portions of BM medium so that the final concentration of each compound (in $\mu \mathrm{g} . / \mathrm{ml}$.) was DL-leucine, DL-isoleucine, DL-valine, DL-threonine, L-arginine, L-cystine, DL-methionine, DL-tryptophan, DL-serine, 50; L-glutamic acid, 200 ; L-lysine, 1000; glycine, 1000.

Sterilization. Solutions of methionine and serine were filtered through Ford SB pads. Sugar solutions, BM medium and solutions of the other amino acids were autoclaved separately at $15 \mathrm{lb} . / \mathrm{in}^{2}$ for $10 \mathrm{~min} .\left(121^{\circ}\right)$.

Incubation. Culture flasks, maintained at $28^{\circ}$ or $35^{\circ} \pm 0 \cdot 1^{\circ}$ as required, were shaken in a Gallenkamp Metabolic Shaker (reciprocal) at 200 strokes (2.5 cm. amplitude)/min. This effected some degree of aeration and also maintained the organisms in suspension.

Auxanographic tests. A technique similar to that described by Pontecorvo (1949) was used. Korean agar (2 g.) was washed by repeated suspension in fresh portions (1 l.) of de-ionized glass-distilled water during the day before use. After standing in water overnight the agar was washed once more, drained of excess water, mixed with BM medium (glucose omitted) and autoclaved $\left(121^{\circ} ; 15 \mathrm{~min}\right.$.). Glucose (or 
maltose) and amino acids as required were added aseptically to the cooled mixture $\left(37-42^{\circ}\right)$. This was then inoculated with the calculated volume of washed suspension of organism. After thoroughly mixing, portions $(10 \mathrm{ml}$.) were quickly run into Petri dishes, allowed to set, and then dried in an inverted position for $1 \mathrm{hr}$ at $35^{\circ}$. Each plate was then overlaid with $5 \mathrm{ml}$. of an aqueous solution of washed agar $(2 \%, w / v)$, and dried similarly. Compounds to be tested were applied to this top layer in very small quantities at well-separated marked positions. Not more than six compounds were applied to dishes of $8.5 \mathrm{~cm}$. diameter.

Replica plating. The technique has been described in detail by Lederberg \& Lederberg (1952).

\section{RESULTS}

Dissociated culture. The fimbriate edges of colonies which developed after repeated subculture of Bacillus cereus $\mathrm{CN} 753$ on nutrient agar at $28^{\circ}$ are shown in Pl. 1, fig. 2. Such a culture will be referred to as a dissociated culture; for comparison the appearance of colonies immediately on reconstitution from freeze-dried specimen is shown in $\mathrm{Pl}$. 1 , fig. 1.

Minimal amino acid requirements for growth at $28^{\circ}$. Auxanographic tests with BM medium and glucose as carbon energy source showed that at $28^{\circ}$ the culture did not grow when ammonium ion was the sole source of nitrogen. Of sixteen common amino acids tested singly only L-glutamic acid or L-aspartic acid supported good growth (Pl. 1, fig. 3). Subsequent tests in liquid BM medium confirmed these results and indicated that a concentration of $1000 \mu \mathrm{g}$. L-glutamate/ml. was optimal. Growth response to $L$-aspartate under the same conditions was erratic. At best, only thin, clumped growth was obtained with L-aspartate at $250 \mu \mathrm{g} . / \mathrm{ml}$.

The effect of lysine + glycine mixture on growing organisms. The effect of these amino acids was first seen during an attempt to determine auxanographically whether or not minimal amino acid requirements varied with the carbon energy source supplied. A series of auxanographs containing pure glucose showed growth only around L-glutamic acid and L-aspartic acid; effects on growth due to the presence of $\mathrm{L}$-lysine and glycine were not demonstrable. However, in a replicate series, the overall growth which resulted from the chance use of a sample of impure maltose (subsequently shown to be contaminated by several amino acids) made it possible to observe, in the particular plate bearing L-lysine and glycine in close proximity, the development of a clear zone between the points at which each had been applied. Within $48 \mathrm{hr}$ after completion of the clearing, comparatively large colonies had developed in the clear zone. The final appearance of such a plate is shown in Pl. 1, fig. 4. Since overall growth was followed by clearing the effect is referred to as lysis rather than as inhibition, although proof of this is wanting. Growth of the dissociated culture, or of certain of the separated variants in repeat tests, did not consistently demonstrate the necessity for the presence of glycine, in addition to L-lysine, for lysis to occur. The shape assumed by a lysed area varied, therefore, between a biconvex area often situated exactly between the separate points of application of lysine and glycine, and a circle surrounding the lysine or lysine+ glycine applications. Lysis was never detected when only glycine was present. The apparent necessity of both amino acids is shown in Pl. 1, fig. 5 .

Prevention of the lytic effect. Plates of BM medium agar containing impure maltose (1 mg./ml.), L-lysine (1 mg./ml.), and glycine (1 mg./ml.), and seeded with the 
dissociated culture, were poured, dried as usual, and then a variety of compounds touched on at separate points. Protection against the lytic effect was shown by the development of dense growth around the active compound. The remaining area of each plate was clear except for a general scatter of a few large colonies of the resistant variants ( $\mathrm{PI} 1$, fig. 6). Compounds having this 'protective' property were the following: L-aspartic acid, L-cystine, L-cysteine, L-histidine, L-glutamine, L-glutamic acid, DL-leucine, DL-isoleucine, L-methionine, L-tyrosine, DL-threonine, DL-valine, DL-norvaline. Those which did not protect were: L- $\alpha$-alanine, $\beta$-alanine, L-arginine, DL- $\alpha$-amino- $n$-butyrate, DL- $\beta$-amino- $n$-butyrate, $\mathrm{DL}$ - $\gamma$-amino- $n$-butyrate, L-hydroxyproline, DL-serine, DL- $\beta$-phenylalanine, DL-tryptophan, L-proline, DLpenicillamine, adenosine, guanine, xanthine, orotic acid, hypoxanthine, thymine, uracil, thiamine, nicotinamide, Ca-D-pantothenate, $p$-aminobenzoic acid, pteroylglutamic acid, pyridoxamine, riboflavine.

\section{Separation of variants sensitive and resistant to mixtures of lysine and glycine}

\section{Lysine-glycine resistant variant (LR 1 )}

An inoculum taken from a colony of Bacillus cereus cN 753 having fimbriate edges was grown in nutrient broth, the organisms washed and then inoculated into cooled molten medium consisting of BM medium (glucose omitted), impure maltose (1\%) and agar (2\%). Plates were poured, dried as usual, and crystals of glycine and $\mathrm{L}$-lysine placed together at marked positions, and the plates then incubated at $28^{\circ}$. Six of the resistant colonies which developed within a lysed area were removed and macerated in $1 \mathrm{ml}$. sterile nutrient broth. This suspension was then inoculated into $20 \mathrm{ml}$. sterile broth and incubated at $28^{\circ}$ on a shaker for $18 \mathrm{hr}$. The organisms were harvested and a washed suspension of just perceptible turbidity prepared. Tenfoldserial dilutions were made from this, $0.05 \mathrm{ml}$. of each dilution spread over the surface of a separate nutrient agar plate and the plates incubated at $28^{\circ}$ overnight. A plate bearing 50-100 well-separated colonies was replica plated in duplicate on BM medium agar containing impure maltose $(1 \%)+\mathrm{L}-$ lysine $(1 \mathrm{mg} . / \mathrm{ml}$. $)+$ glycine (1 mg./ml.), and also on nutrient agar. To obtain colonies of reasonable size it was necessary to incubate the $\mathbf{B M}$ medium plates for four days at $28^{\circ}$. Nutrient agar plates required only overnight incubation at $28^{\circ}$ and were then retained at $4^{\circ}$. All the colonies on the nutrient agar replica appeared on the selective defined medium; some had an entire edge, while others had such a pronounced lobate edge that they assumed a satellite appearance (Pl. 2, fig. 7). Since the form of the lobate colony was retained on duplicate plates it seemed that it was not the result of a mechanical artifact. Probably the colonial form represented the resistant portions of a colony remaining after those portions sensitive to lysine + glycine had lysed. Isolates were obtained by picking from a single colony of each type. Organisms grown from these, washed and seeded into BM medium containing impure maltose + glycine + agar, showed no lysis around applied crystals of lysine. Similar plates seeded with the dissociated culture of $\boldsymbol{B}$. cereus $\mathrm{CN} \mathbf{7 5 3}$ showed the typical lytic effect. A freezedried stock was prepared from the smooth-edge type and designated LR 1 .

Lysine-glycine sensitive variant (LS)

Using an inoculum of washed cells grown from a fimbriate colony of $B$. cereus CN 753, replica plates were again prepared as described above but with BM medium 
agar + impure maltose. Prior to incubation, crystals of L-lysine and glycine were placed together on the plates at precisely located positions. The plates were then incubated and colonies sought which had lysed (in areas close to the lysine + glycine applications) and remained so upon extended incubation ( 1 week at $28^{\circ}$ ). Inocula taken from corresponding colonies on the nutrient agar replica were then replica plated repeatedly until many colonies present on the nutrient agar replica remained completely absent from the selective replica. When this was attained, a colony present on the nutrient agar plates but absent from the corresponding position on selective replicas was then picked and grown in nutrient broth to provide organisms from which a freeze-dried stock of the sensitive variant, Ls, was prepared.

An auxanographic comparison of the purity of inocula grown from variants LS and LR 1 is shown in Pl. 2, figs. 8, 9. Subsequent work indicated that variant Ls remained as pure as on isolation but LR 1 seemed to develop an increasing amount of the sensitive component. This was manifest as a definite thinning of the opacity of plates inoculated with LR 1 in the vicinity of superimposed crystals of lysine and glycine. A suitable plate, inoculated with LR 1 and bearing L-lysine and glycine at one site, was therefore selected and one matt colony and one smooth colony picked from the surface at a thinning area. Each colony was suspended in a small volume of saline and then streaked on to BM medium agar containing impure maltose $(1 \%)+\mathrm{L}-$ lysine $(1 \mathrm{mg} . / \mathrm{ml}$.) + glycine ( $1 \mathrm{mg} . / \mathrm{ml}$.). Growth was slow, but after 7 days at $28^{\circ}$ single colonies were large enough to transfer to nutrient broth; after overnight growth at $28^{\circ}$ the crops were freeze-dried to provide stocks designated LR $2 / \mathrm{R}$ and $L R 2 / s$, respectively.

\section{Further resolution of variants $L S$ and $L R 2 / S$}

During subsequent work it was noticed that on plating the crops obtained by growing these variants in chemically defined (CDM) medium some of the colonies maintained an entire edge, whereas others quickly developed a fimbriate edge. Microscopically each type of colony consisted of large Gram-positive rods; macroscopically the colonial appearances were not sufficiently dissimilar to suggest contamination. An extreme example is shown in Pl. 2, fig. 10: all colonies from one culture vessel are entire-edged while almost all of those from another are fimbriate though both culture flasks were inoculated with variant $\mathrm{Ls}$.

Subcultures on nutrient agar were made from the centre of an entire-edge colony and the periphery of a fimbriate type, and by repeated subculture of young colonies $\left(18 \mathrm{hr}\right.$ at $\left.28^{\circ}\right)$ cultures which retained their entire and fimbriate colonial characteristics were obtained (Pl. 3, figs. 11-14). Freeze-dried stocks were prepared from crops grown in nutrient broth and designated variants LS/S, LS/R, LR 2/SS, LR 2/SR.

\section{Derivation of variant $L R / V R$}

Routine plating of variant $L R 1$ revealed the existence of a minute proportion of colonies which developed exaggerated fimbriate characteristics. One was picked, repeatedly subcultured on nutrient agar and a crop then freeze-dried for stock. The relationship of the variants to each other is shown in Fig. 1, and their colonial appearances in Pl. 3, figs. 11-14. 


\section{Investigations with the separated variants}

Re-typing. The use of infra-red spectrophotometry as an aid in the identification of bacteria has been suggested by many workers (Stevenson \& Bolduan, 1952; Levine, Stevenson, Chambers \& Kenner, 1953; Riddle et al. 1956), though several consider it of limited application. The work of Blackwood \& Epp (1957), and Haynes

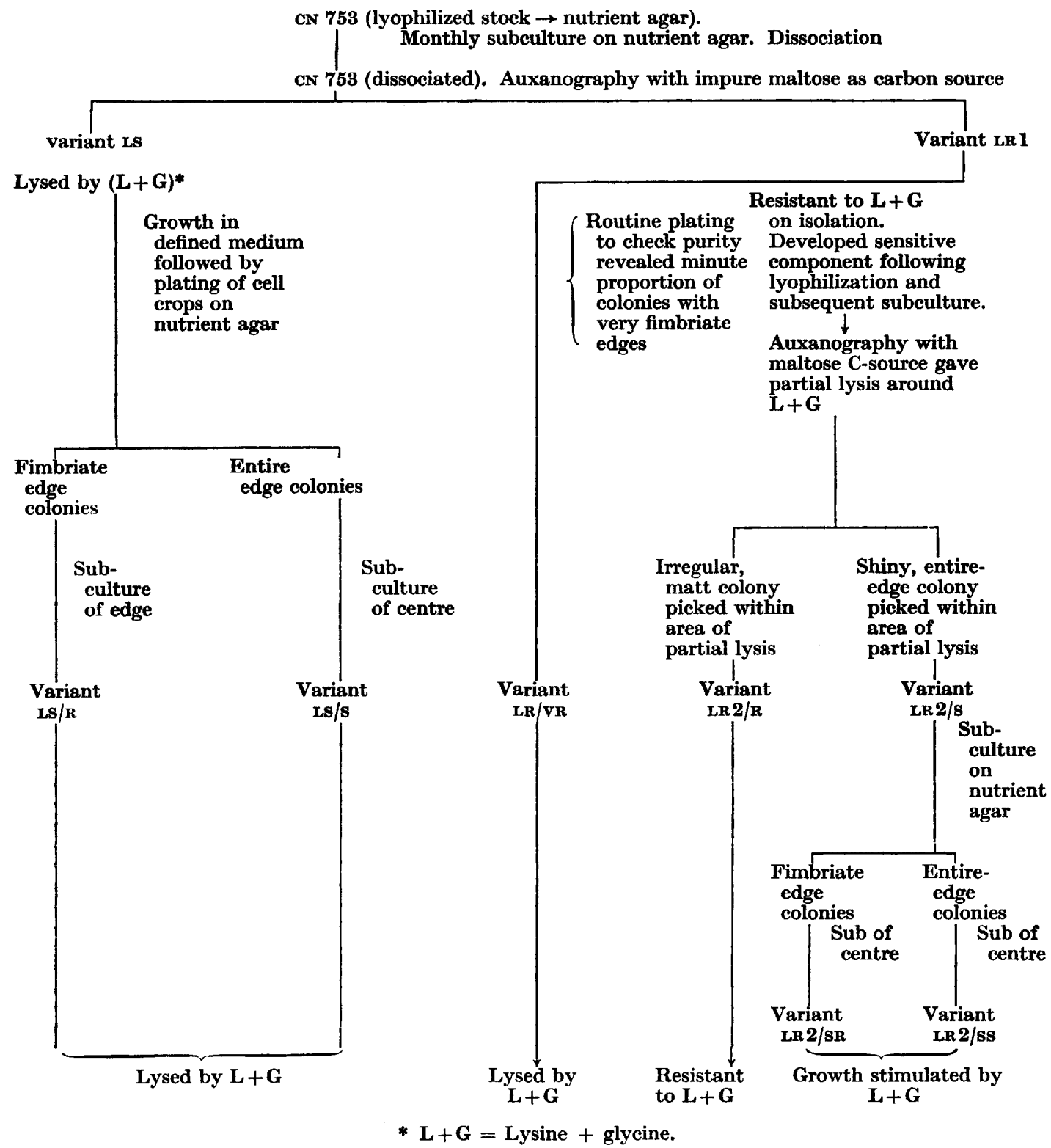

Fig. 1. The relationship and derivation of variants isolated from the dissociated culture of Bacillus cerens CN 753 . 
et al. (1958), however, indicated that the technique was useful for characterizing members of the genus Bacillus. By virtue of their content of poly- $\beta$-hydroxybutyric acid, which absorbs strongly at $5 \cdot 7 \mu$, whole organisms of members of this genus are endowed with a characteristic ' $5 \cdot 7 \mu$-type' absorption spectrum (Haynes et al. 1958) when grown under appropriate conditions. Whole organisms of the variants and of the dissociated culture, grown in nutrient broth $(0.2 \%$ glucose $)$ at $28^{\circ}$, had identical infra-red spectra, each with strong absorption band at $5 \cdot 7 \mu$.

Tests based upon those suggested by Smith et al. (1952) showed that each isolate exhibited biochemical characteristics typical of Bacillus cereus, viz.: gelatin liquefaction + ; casein hydrolysis + ; starch hydrolysis + ; anaerobic growth (Robertson's broth) + ; aerobic growth ( $\mathrm{pH} \mathrm{6 \cdot 0)} \mathrm{+;} \mathrm{acetylmethylcarbinol} \mathrm{formation} \mathrm{+} \mathrm{;} \mathrm{methy-}$ lene-blue reduction - ; lecithinase (egg-yolk reaction) + ; methyl red test + ; indole formation - ; formation of nitrite from nitrate + ; utilization of citrate - ; urease formation - ; bromocresol purple milk, alkaline with peptonization; utilization of carbohydrates. (a) Inorganic medium: glucose, growth with formation of acid; D-xylose and L-arabinose, no growth, no acid formation. (b) Organic medium: glucose, growth with acid formation; growth and acid formation in the presence of L-arabinose or D-xylose was atypical.

Nutritionally each isolate required a supply of preformed amino acids for growth in defined medium but a supply of growth factors of the vitamin B complex was not necessary. These requirements are in agreement with those found for Bacillus cereus, by Knight \& Proom (1950).

$S-R$ classification. Impression preparations of young $(9 \mathrm{hr})$ colonies were made according to the method of Klieneberger \& Smiles (1942), but stained with tannic acid crystal violet according to Robinow (1947) to reveal the cell-walls. On the basis of the microscopic appearance of the organisms and their arrangement within the colonies each variant appeared to represent a rough $(R)$ type (Bisset, 1938, 1948) (Pl. 3, figs. 11-15).

At $28^{\circ}$ on nutrient agar entire-edge types, namely, variants of $L S / S$ and LR2/ss produced colonies having only a suggestion of medusa-head structure; this became a little more pronounced at $35^{\circ}$. The remainder of the variants produced typical medusa-head colonies at both temperatures.

Minimal amino acid requirements. These were determined auxanographically with glucose as carbon energy source and for ease of comparison are shown in Table 1. The different auxanographic responses to lysine, glycine, and lysine + glycine are compared in Pl. 4, figs. 16-21. Lysine was not replaceable by cadaverine, putrescine, histamine, benzylamine or ethanolamine, when tests were carried out with variant $\mathrm{LS} / \mathrm{s}$, though partial lysis occurred when lysine was replaced by 11-aminoundecanoic acid, w-aminocaprylic acid, or $\alpha$-aminopimelic acid.

The growth response of the dissociated culture and of each of the variants at $28^{\circ}$, in BM medium containing $500 \mu \mathrm{g}$. L-glutamate/ml., is shown in Fig. 2.

The effect of temperature on growth and amino acid requirements. In a defined medium consisting of BM, glucose and L-glutamate $(1 \mathrm{mg} . / \mathrm{ml}$.) washed bacteria of the dissociated culture grew heavily at $28^{\circ}$ but not at all at $35^{\circ}$. Growth of unwashed bacteria in the same medium at $35^{\circ}$ showed that proliferation at this temperature was, in fact, possible, and that one or more factors present in broth were essential for growth. The latter could not be replaced by a second amino acid, or purines, 
pyrimidines, nucleotides and factors of the vitamin B-complex, singly or in admixture. The compounds concerned were in fact a group of amino acids, namely: DL-leucine, DL-threonine, L-arginine, DL-isoleucine and DL-valine. Incorporation of each of these, at $50 \mu \mathrm{g} . / \mathrm{ml}$. into BM containing glucose and L-glutamic acid, together with L-lysine, glycine and L-cysteine, present in the medium of Johnson (1961), resulted in rapid heavy growth of washed bacteria of the dissociated culture and of each variant at $35^{\circ}$. Omission of the amino acids singly showed that for

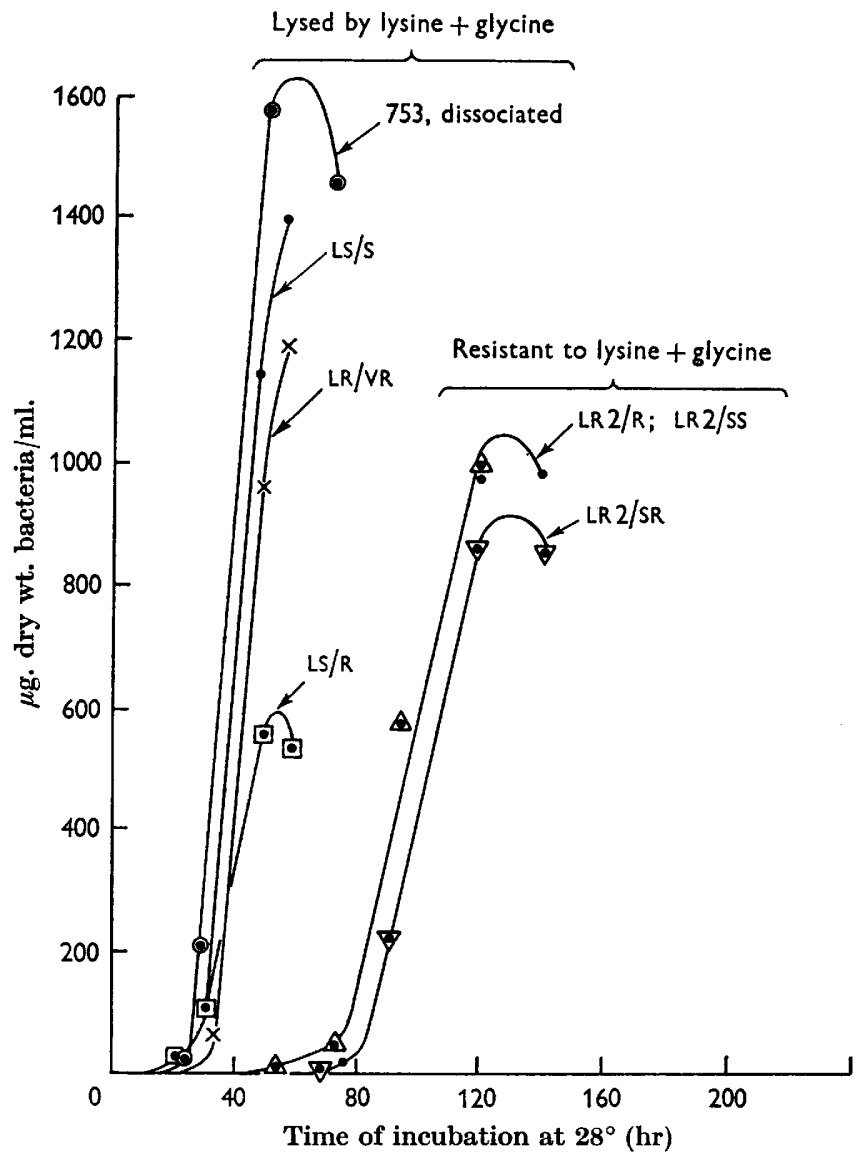

Fig. 2. Growth at $28^{\circ}$ of the dissociated culture of Bacillus cereus $\mathrm{CN} 753$ and each of the variants in defined medium (salts + glucose) containing L-glutamic acid (500 $\mu \mathrm{g} . / \mathrm{ml}$.) as sole nitrogen source.

growth of variant $\mathrm{LS} / \mathrm{s}$ (the only variant investigated) at $35^{\circ}, \mathrm{DL}$-isoleucine and L-cysteine were not essential, DL-serine and glycine were stimulatory, L-lysine inhibitory, and the remaining amino acids, namely, L-glutamic acid, DL-leucine, DL-valine, DL-threonine and L-arginine, essential. 
Table 1. Minimal amino acid requirements of variants of Bacillus cereus CN 753 tested auxanographically in solid defined medium containing salts and glucose

\begin{tabular}{|c|c|c|c|c|c|c|c|}
\hline \multirow{2}{*}{$\begin{array}{c}\text { Amino acid } \\
\text { incorporated } \\
\text { in basal medium }\end{array}$} & \multirow{2}{*}{$\begin{array}{c}\text { Amino acid applied } \\
\text { to surface of solid } \\
\text { medium }\end{array}$} & \multicolumn{6}{|c|}{ Growth-response variant } \\
\hline & & $\mathbf{L S} / \mathbf{s}^{1 *}$ & $\mathbf{L S} / \mathbf{R}^{2 *}$ & $\operatorname{LR} 2 / \mathrm{SS}^{3 *}$ & $\mathrm{LR} 2 / \mathrm{SR}^{4 *}$ & $\operatorname{LR} 2 / \mathbf{R}^{5 *}$ & $\mathrm{LR} / \mathrm{VR}^{\mathrm{B*}}$ \\
\hline None & $\begin{array}{l}\text { L- } \alpha \text {-Alanine } \\
\text { L-Aspartic acid } \\
\text { L-Cysteine-HCl } \\
\text { Glycine } \\
\text { L-Lysine (HCl) } \\
\text { L-Glutamic acid } \\
\text { L-Proline } \\
\text { L-Histidine } \\
\text { DL-Tryptophan } \\
\text { DL-Serine } \\
\text { DL-Leucine } \\
\text { DL-iso-Leucine } \\
\text { DL-Threonine } \\
\text { DL-Methionine } \\
\text { DL-Valine } \\
\text { L-Arginine (HCl) }\end{array}$ & $\begin{array}{c}- \\
+ \\
- \\
- \\
++ \\
+ \\
++ \\
- \\
- \\
- \\
+ \\
+ \\
+ \\
+\end{array}$ & $\begin{array}{l}- \\
- \\
- \\
- \\
++ \\
- \\
- \\
- \\
- \\
- \\
- \\
- \\
-\end{array}$ & $\begin{array}{c}\overline{+} \\
- \\
- \\
- \\
+++ \\
\overline{+} \\
++ \\
- \\
- \\
- \\
- \\
- \\
- \\
-\end{array}$ & $\begin{array}{c}\overline{+}+ \\
- \\
- \\
\overline{+} \\
+ \\
+\overline{+}+ \\
- \\
- \\
- \\
- \\
- \\
- \\
-\end{array}$ & $\begin{array}{l}- \\
- \\
- \\
- \\
- \\
- \\
- \\
- \\
- \\
- \\
- \\
-\end{array}$ & $\begin{array}{l}- \\
(+) \\
- \\
- \\
+ \\
+ \\
+ \\
- \\
- \\
- \\
- \\
- \\
-\end{array}$ \\
\hline L- $\propto$-Alanine & $\begin{array}{l}\text { L-Aspartic acid } \\
\text { L-Cysteine (HCl) } \\
\text { Glycine } \\
\text { L-Lysine (HCl) } \\
\text { L-Glutamic acid }\end{array}$ & $\begin{array}{l}+ \\
- \\
- \\
+\end{array}$ & $\begin{array}{l}(+) \\
- \\
- \\
(+)\end{array}$ & $\begin{array}{c}+++ \\
- \\
- \\
+++\end{array}$ & $\begin{array}{c}+t+ \\
- \\
- \\
++t\end{array}$ & $\begin{array}{c}- \\
+ \\
- \\
+\end{array}$ & $\begin{array}{l}++ \\
(+) \\
- \\
- \\
++\end{array}$ \\
\hline L-Aspartic acid & $\begin{array}{l}\text { L-Cysteine (HCl) } \\
\text { Glycine } \\
\text { L-Lysine (HCl) } \\
\text { L-Glutamic acid }\end{array}$ & $\begin{array}{l}\ddagger \\
\vdots \\
\ddagger \\
\ddagger\end{array}$ & $\begin{array}{l}- \\
- \\
-\end{array}$ & $\begin{array}{l}\ddagger \\
\ddagger \\
\ddagger \\
\ddagger\end{array}$ & $\begin{array}{l}\ddagger \\
\ddagger \\
\ddagger \\
\ddagger\end{array}$ & $\begin{array}{c}++ \\
- \\
- \\
+\end{array}$ & $\begin{array}{l}(+) \\
- \\
- \\
++\end{array}$ \\
\hline L-Cysteine (HCl) & $\begin{array}{l}\text { Glycine } \\
\text { L-Lysine (HCl) } \\
\text { L-Glutamic acid }\end{array}$ & $\underline{-}$ & $\begin{array}{l}- \\
+ \\
++\end{array}$ & $\overline{-}$ & $\begin{array}{c}- \\
+\overline{+}+\end{array}$ & $\frac{-}{++}$ & $\frac{-}{+}$ \\
\hline L-Lysine (HCl) & $\begin{array}{l}\text { L-Lysine (HCl) } \\
\text { L-Glutamic acid }\end{array}$ & $\overline{+}$ & + & $+\overline{+}+$ & $+\overline{+}+$ & $\overline{-}$ & - \\
\hline L-Lysine (HCl) & L-Glutamic acid & $+t$ & ++ & +++ & $+t+$ & - & ++ \\
\hline
\end{tabular}

$(+)$ indicates faint growth; + , poor growth; ++ , medium growth; +++ , heavy growth.

* Minimum incubation period at $28^{\circ}$ for unequivocal response; $1,23 \mathrm{hr} ; 2,113 \mathrm{hr} ; 3,88 \mathrm{hr}$; $4,85 \mathrm{hr} ; 5,134 \mathrm{hr} ; 6,113 \mathrm{hr}$.

$\dagger$ Growth in admixture i.e. in centre of plate.

$\ddagger$ Heavy overall growth.

\section{DISCUSSION}

The results presented show that maintenance of the particular culture of Bacillus cereus used, by repeated subculture on nutrient agar, is undesirable. After many transfers it is possible to observe that a heterogeneous population becomes established since the colonial morphology alters. The physico-chemical and gross biochemical characteristics of pure cultures of the different variants however remain essentially those of $B$. cereus though their ability to utilize L-arabinose and Dxylose is anomalous. Recovery of the same morphological types after freeze-drying reconstitution and subculture, and also after prolonged ( 5 days) incubation in defined media at $28^{\circ}$ or $35^{\circ}$ suggests that each of the variants is relatively stable.

The pattern of nutritional requirements, namely, preformed amino acids essential, B vitamins not essential, remains typically that of Bacillus cereus, though 
individual differences in amino acid requirements are shown. Variant Ls/s probably represents the major proportion of the population of the original dissociated culture since it grows most rapidly and is least exacting, i.e. it will grow in the presence of a single amino acid of the group, glutamic acid, aspartic acid, proline, histidine, threonine or methionine. The other variants are progressively more exacting in that either the number of amino acids which can support growth is fewer (one only for variant $L S / R$, three for $L R / V R$ ) or that at least two amino acids together are essential (variant LR 2/R). These facts presumably reflect differences in the equilibrium positions of metabolic reactions amounting, in some instances, to deficient formation of certain essential compounds. Such variants thus become less well equipped to establish themselves in a mixed population containing variant $\mathrm{Ls} / \mathrm{s}$ organisms. The fact that only one or two colonies of variant LR/VR can ever be seen when the undissociated culture of B. cereus $\mathbf{C N} 753$ is plated possibly bears this out. The number of colonies representing the more exacting (lysine resistant) variants, namely, LR 2/R, $\mathrm{LR} 2 / \mathrm{sS}, \mathrm{LR} 2 / \mathrm{SR}$, in lytic areas of auxanographic plates is also so much smaller than the total number of bacteria originally present per unit volume, that their combined numbers can represent only a fraction of the total population of the inoculum.

That the ability of the dissociated culture to grow at $35^{\circ}$ in the presence of additional amino acids is an expression of the proliferation of a variant favoured by the higher incubation temperature seems unlikely since all the variants isolated show the same effect and are recoverable from cultures grown at $35^{\circ}$. Since growth occurs at $28^{\circ}$ in the presence of glutamate only, and at $35^{\circ}$ only when additional amino acids are supplied, it appears that at the higher temperature the ability of the bacteria to derive necessary amino acids from glutamate is impaired.

From a consideration of colonial morphology and amino acid requirements it seems that while the pairs of variants $\mathrm{LS} / \mathrm{s}, \mathrm{LR} 2 / \mathrm{ss}$, and LR2/R, LR/VR represent extremes (the former being least exacting and barely maintaining rough (R) colonial characteristics, and the latter more exacting and distinctly R-types), variants LR2/SR and LS/R might well represent intermediate types, or populations of incompletely separated variants. Thus nutritionally these retain less exacting characteristics, due perhaps to the presence of an Ls/s-type, but morphologically the irregular edges of their colonies may represent the proliferation of an LR 2/R-type.

The variants fall into two groups on the basis of growth rate and lysine sensitivity, the faster growing ( $\mathrm{LS} / \mathrm{s}, \mathrm{LS} / \mathrm{R}, \mathrm{LR} / \mathrm{VR})$ being sensitive to L-lysine and the slower (LR 2/R, LR 2/SS, LR 2/SR) resistant. Although elaboration of lytic factors by members of the genus Bacillus is very well known (Waksman, 1945) the nature of the factors is often less well established. Smith et al. (1952) considered the lytic activity present in their cultures of $B$. cereus due to bacteriophage, whilst Nomura \& Hosoda (1956) and Ivanovics \& Alföldi (1957) described, respectively, the formation of an autolytic enzyme by $\boldsymbol{B}$. subtilis, and a proteinaceous antibacterial lytic substance (megacin) by $\boldsymbol{B}$. megaterium. Reports of the lytic effect of cysteamine and its close derivatives for members of the genus Bacillus have been published by Weinberg, Saz \& Pilgren (1958) and Judith \& Weinberg (1959). In the former paper several mechanisms were suggested which would provide an explanation of the lytic effects observed and equally of the lytic effect obtained with L-lysine in the present work. The fact that 11-aminoundecanoic acid, $\omega$-aminocaprylic acid, or $\alpha$-aminopimelic acid can replace L-lysine to some extent suggests, on the basis of similarity of chemical 
structure, that these compounds interfere with formation and/or the incorporation of $\alpha$ - $\epsilon$-diaminopimelic acid into the cell wall.

My thanks are due to Miss B. Rawlings and Mr R. Gower for their willing and meticulous technical assistance, and Dr A. W. Phillips for much useful advice.

\section{REFERENCES}

Bisser, K. A. (1938). The structure of 'rough' and 'smooth' colonies. J. Path. Bact. 47, 223.

Bisset, K. A. (1948). The cytology of smooth and rough variation in bacteria. J. gen. Microbiol. 2, 83.

Blackwood, A. C. \& EPp, A. (1957). Identification of $\beta$-hydroxybutyric acid bacterial cells by infra-red spectrophotometry. J. Bact. 74, 266.

Braun, W. (1947). Bacterial dissociation. Bact. Rev. 11, 75.

Brewer, C. R., McCullough, W. G., Mills, R. C., Roessler, W. G., Herbst, E. J. \& Howe, A. F. (1946). Studies on the nutritional requirements of Bacillus anthracis. Arch. Biochem. 10, 65.

Campbeli, L. L. \& Sniff, E. E. (1959). Folic acid requirement of Bacillus coagulans. J. Bact. 78, 267.

Cleverdon, R. C., Pelczar, M. J. Jun. \& Doetsch, R. N. (1949a). Vitamin requirements of Bacillux coagulans. J. Bact. 58, 113.

Cleverdon, R. C., Pelczar, M. J. Jun. \& Doetsch, R. N. (1949b). The vitamin requirements of stenothermophilic aerobic sporogenous bacilli. J. Bact. 58, $\mathbf{5 2 3}$.

Demarn, A. L. (1958). Minimal media for quantitative studies with B. subtilis. J. Bact. $75,517$.

Dutky, S. R. (1947). Preliminary observations on the growth requirements of Bacillus popilliae Dutky, and Bacillus lentimorbus Dutky. J. Bact. 54, 257.

Haynes, W. C., Melvin, E. H., Locke, J. M., Glass, C. A., Sentr, F. R. (1958). Certain factors affecting the infrared spectra of selected microorganisms. Appl. Microbiol. 6, 298.

Ivanovics, G. \& AlFöLdi, L. (1957). Bacteriocinogenesis in Bacillus megaterium. J. gen. Microbiol. 16, 522.

Johnson, R. M. (1961). Large cell stage in the genus Bacillus. J. Bact. 82, 418.

Judith, F. R. \& Weingerg, E. D. (1959). Correlation of amine lysis with species differentiation in the genus Bacillus. J. Bact. 78, 485.

Katznetson, H. (1944). The differentiation of Bacillus polymyxa and Bacillus macerans on the basis of their vitamin requirements. J. Bact. 48, 495.

Katzenelson, H. \& LochHead, A. G. (1947). Nutritional requirements of Bacillus alvei and Bacillus para-alvei. J. Bact. 53, 83.

Katznelson, H. \& Lochiead, A. G. (1948). Nutritional requirements of Bacillus larvae. J. Bact. 55, 763.

KuIENEBERger, E. \& Smines, J. (1942). Some new observations on the developmental cycle of the organism of bovine pleuropneumonia and related microbes. J. Hyg., Camb. $42,110$.

Knight, B. C. J. G. \& Proom, H. (1950). A comparative survey of the nutrition and physiology of mesophilic species in the genus Bacillus. J. gen. Microbiol. 4, 508.

Lederberg, J. \& Lederberg, E. M. (1952). Replica plating and indirect selection of bacterial mutants. J. Bact. 63, 399.

Levine, S., Stevenson, H. J. R., Chambers, L. A. \& Kenner, B. A. (1953). Infra-red 44, 185.

LochHEAd, A. G. (1942). Growth factor requirements of Bacillus larvae, White. J. Bact. spectrophotometry of enteric bacteria. J. Bact. 65, 10.

Moore, W. B. (1963). A culture flask for aerobic micro-organisma. J. lab. Practice, 12, 450.

Nomura, M. \& Hosoda, J. (1956). Nature of the primary action of the autolysin of Bacillus subtilis. J. Bact. 72, 573. 
Ponteconvo, G. (1949). Auxanographic techniques in biochemical genetics. J. gen. Microbiol. 3, 122.

Proom, H. \& Knight, B. C. J. G. (1955). The minimal nutritional requirements of some species in the genus Bacillus. J. gen. Microbiol. 13, 474.

Riddle, J. W., Kabler, P. W., Kenner, B. A., Bordner, R. H., Rochwood, S. W. \& Stevenson, H. J. R. (1956). Bacterial identification by infra-red spectrophotometry. J. Bact. 72, 593.

Rosinow, C. F. (1947). Addendum to 'The Bacterial Cell' by Dubos, R. J. Cambridge, Mass. : Harvard University Press.

Smith, N. R., Gordon, R. E. \& Clakk, F. E. (1952). U.S. Dep. Agric. Monograph no. 16, Washington, D. C.

Stevenson, H. J. R. \& Bolduan, O. E. A. (1952). Infra-red spectrophotometry as a means for identification of bacteria. Science, 116, 111.

TEAs, H. J. (1950). Mutants of Bacillus subtilis that require threonine or threonine+ methionine. J. Bact. 59, 93.

Waksman, S. A. (1945). Microbial Antagonisms and Antibiotic Substances. New York: The Commonwealth Fund.

Weinberg, E. D., Saz, A. H. \& Pilgren, E. Y. (1958). Induction of bacteriolysis by cysteamine and its derivatives. J. gen. Microbiol. 19, 419.

\section{EXPLANATION OF PLATES}

\section{Plate 1}

Fig. 1. Appearance of colonies of undissociated Bacillus cereus cN 753 after reconstitution of freeze-dried stock and cultivation on nutrient agar at $28^{\circ}$ for $18 \mathrm{hr}$.

Fig. 2. As Fig. 1, but after repeated subculture on nutrient agar.

Fig. 3. Auxanographic response of dissociated $B$. cereus CN 753 to L-aspartate (17) and L-glutamate (21), basal salts medium with glucose as carbon energy source.

Fig. 4. As fig. 3, but with impure maltose present instead of glucose, showing overall growth with lysed area (containing resistant colonies) between $L$-lysine (19) and glycine (20).

Fig. 5. As fig. 3, but inoculated with variant $L \mathrm{~s} / \mathrm{s}$ and supplemented with L-methionine (1 $\mu \mathrm{g} . / \mathrm{ml}$.); showing lysed areas between lysine and glycine, and around lysine + glycine.

Fig. 6. Plate containing basal salts medium, impure maltose, $\mathrm{L}$-lysine (1 mg./ml.), glycine (1 mg./ ml.) and seeded with dissociated $B$. cereus $\mathrm{CN} 753$, showing protection against lysis afforded by glycyl-DL-asparagine (10).

\section{Plate 2}

Fig. 7. 'Satellite' appearance of replica plated colonies of Bacillus cereus CN 753 variant LR 1, possibly indicating lysis of contaminant Ls-type bacteria. A few colonies, virtually pure LR-types, show no satellites. Solidified basal salts medium + maltose (impure) $+\mathrm{L}$-lysine (1 mg./ml.) + glycine (1 mg./ml.).

Figs. 8, 9. Auxanographic comparison of the purity of isolates LS (fig. 2) and LR 1 (fig. 3) by their responses to lysine + glycine mixture.

Fig. 10. Plate to check purity of variant $\mathrm{Ls}$ after growth in defined medium. Crops represented pure cultures but a high proportion of colonies developing from bacteria taken from one culture flask (10) show fimbriate edges, while all those from replicate flask (12) show entire edges.

\section{Plate 3}

Appearance of organisms and colonies of variants of Bacillus cereus CN 753 grown on nutrient agar at $28^{\circ}$, for $9 \mathrm{hr}$ (bacteria), $18 \mathrm{hr}$ (colonies). Preparations by the impression technique. Tannic acid crystal violet stain. Magnification: colonies $\times 3 \frac{1}{2}$; organism $\times 1600$.

Fig. 11. Variants LS/S or LR2/ss, showing colonies with entire peripheries.

Fig. 12. Variant $\mathrm{LS} / \mathrm{R}$, showing colonies with irregular peripheries which later become fimbriate.

Fig. 13. Variant LR2/sR, showing colonies with irregular peripheries which later become fimbriate.

Fig. 14. Variant LR/VR, showing pronounced fimbriate peripheries of young colonies.

Fig. 15. Variant LR2/R, showing small colonies with edges which remain irregular. 


\section{Plate 4}

Auxanographic response of variants of Bacillus cereus CN 753 to L-lysine, glycine and L-lysine + glycine.

Fig. 16. Variant $\mathrm{Ls} / \mathrm{s}$ showing lysis around lysine, and lysine + glycine, but not around glycine.

Fig. 17. Variant Ls/R (and LR/VR), Growth is poor but lysis just discernible around lysine and lysine + glycine.

Fig. 18. Variant LR 2/ss; growth increased by lysine.

Fig. 19. Variant LR2/sR; growth increased by lysine. The crescent-shaped areas of thinning growth between $L, G$, and $L, L+G$ applications indicate the presence of a small percentage of sensitive cells.

Fig. 20. Variant LR2/R. Growth unaffected by lysine, glycine, or lysine + glycine.

Fig. 21. Dissociated Bacillus cereus cN 758. Growth lysed by L-lysine and L-lysine+glycine. Resistant colonies developing within the lytic area. 
Journal of General Microbiology, Vol. 40, No. 3

Plate 1

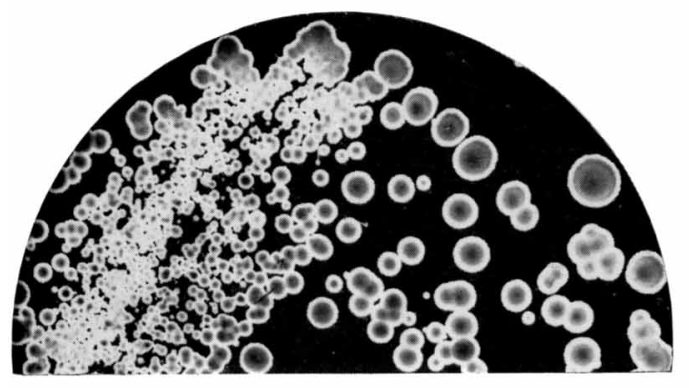

1
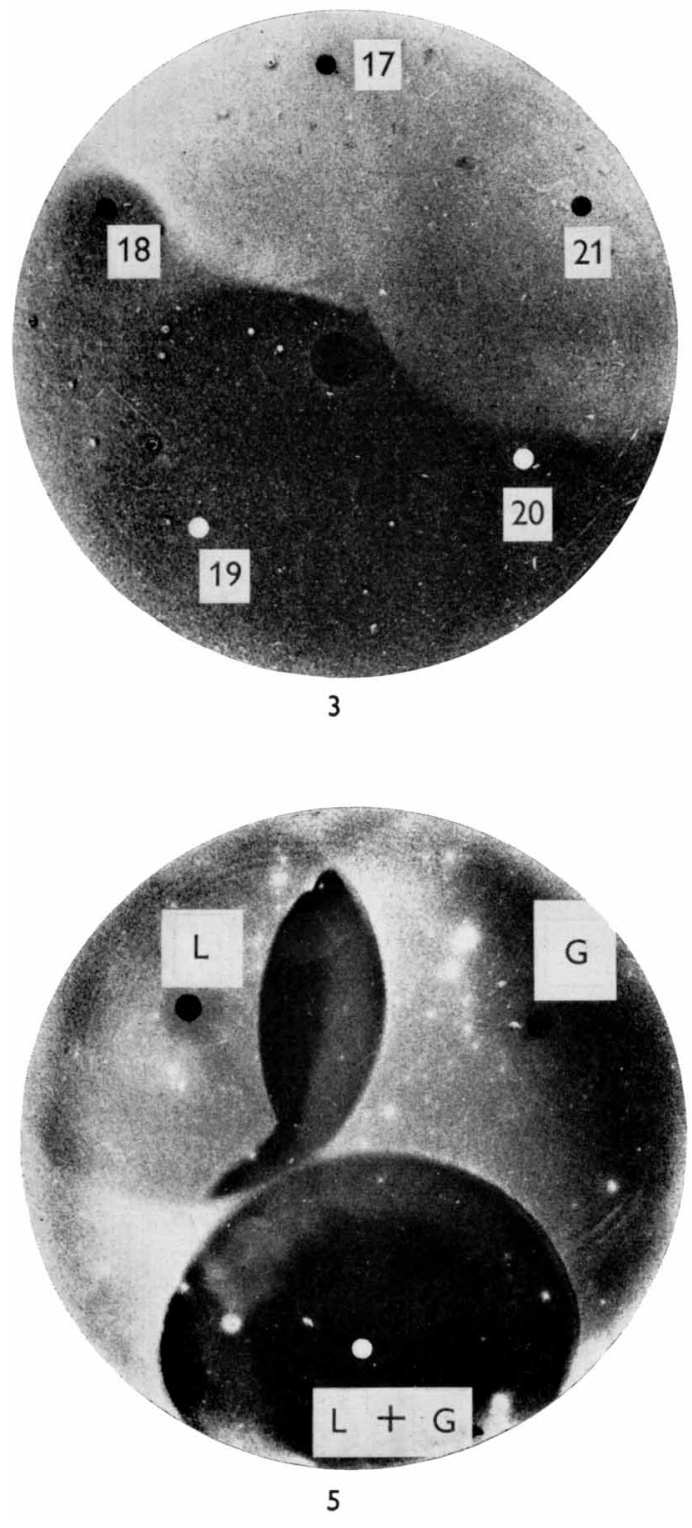

W. B. MOORE

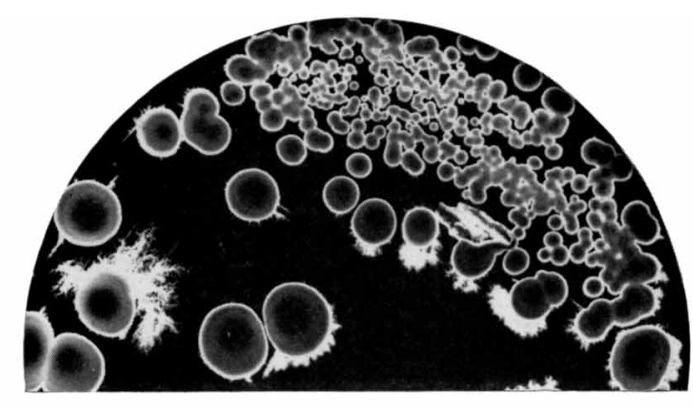

2
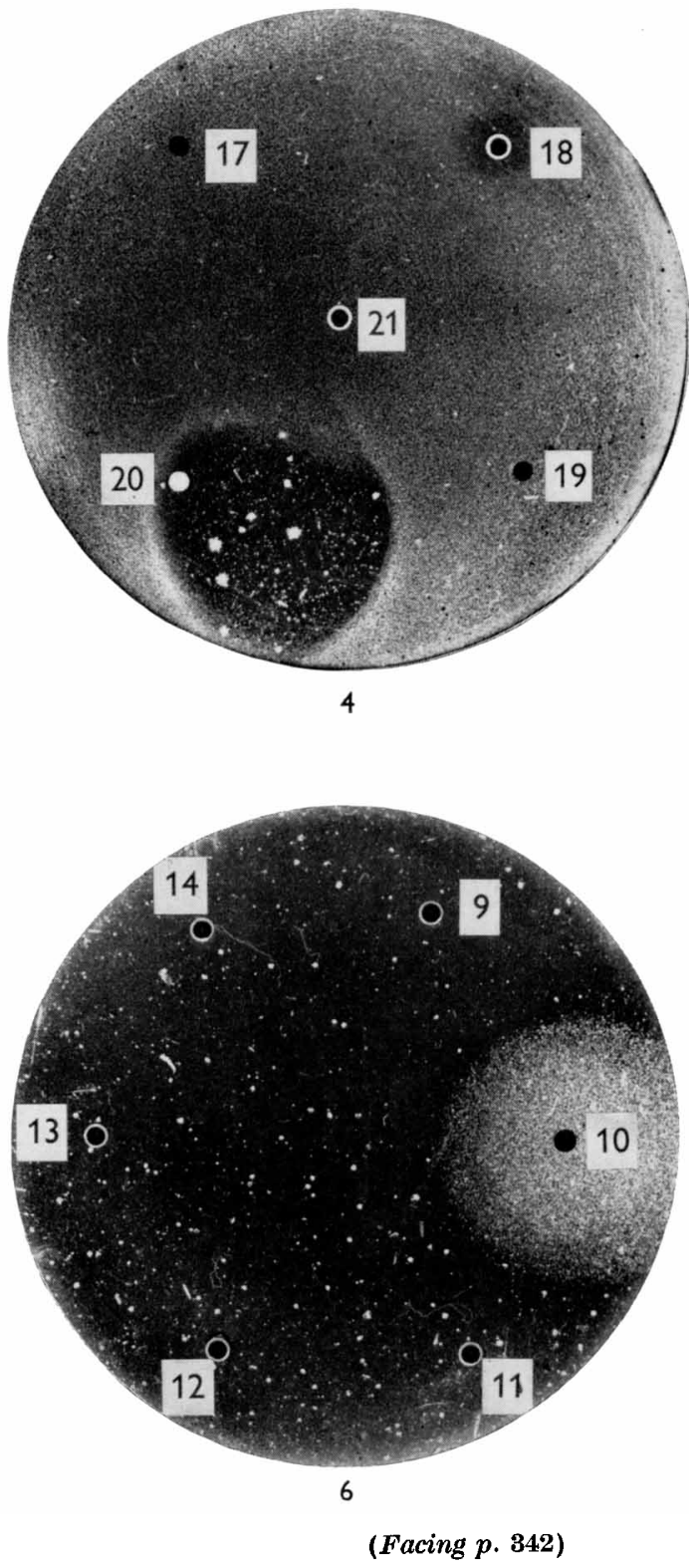

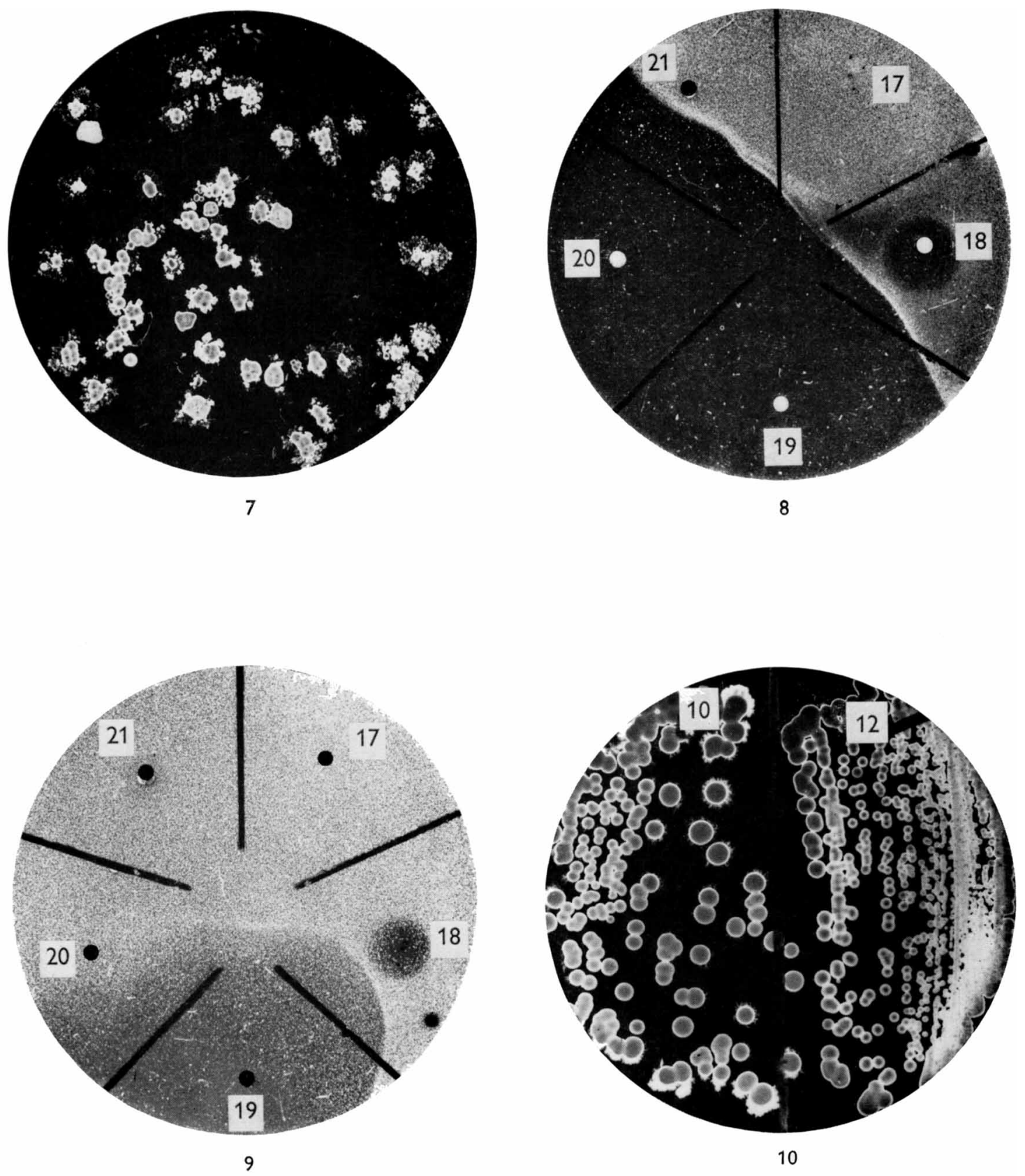

W. B. MOORE 

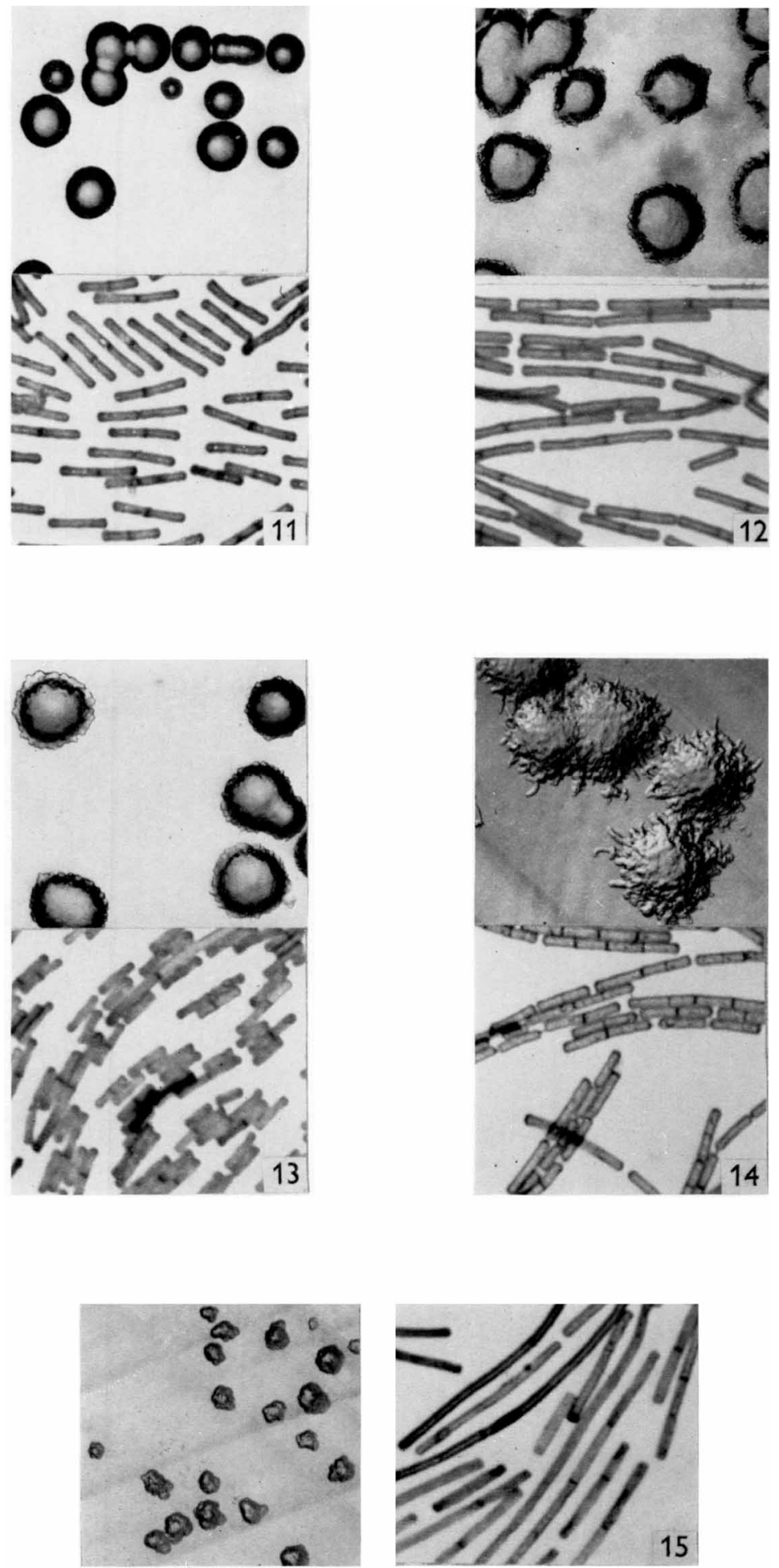

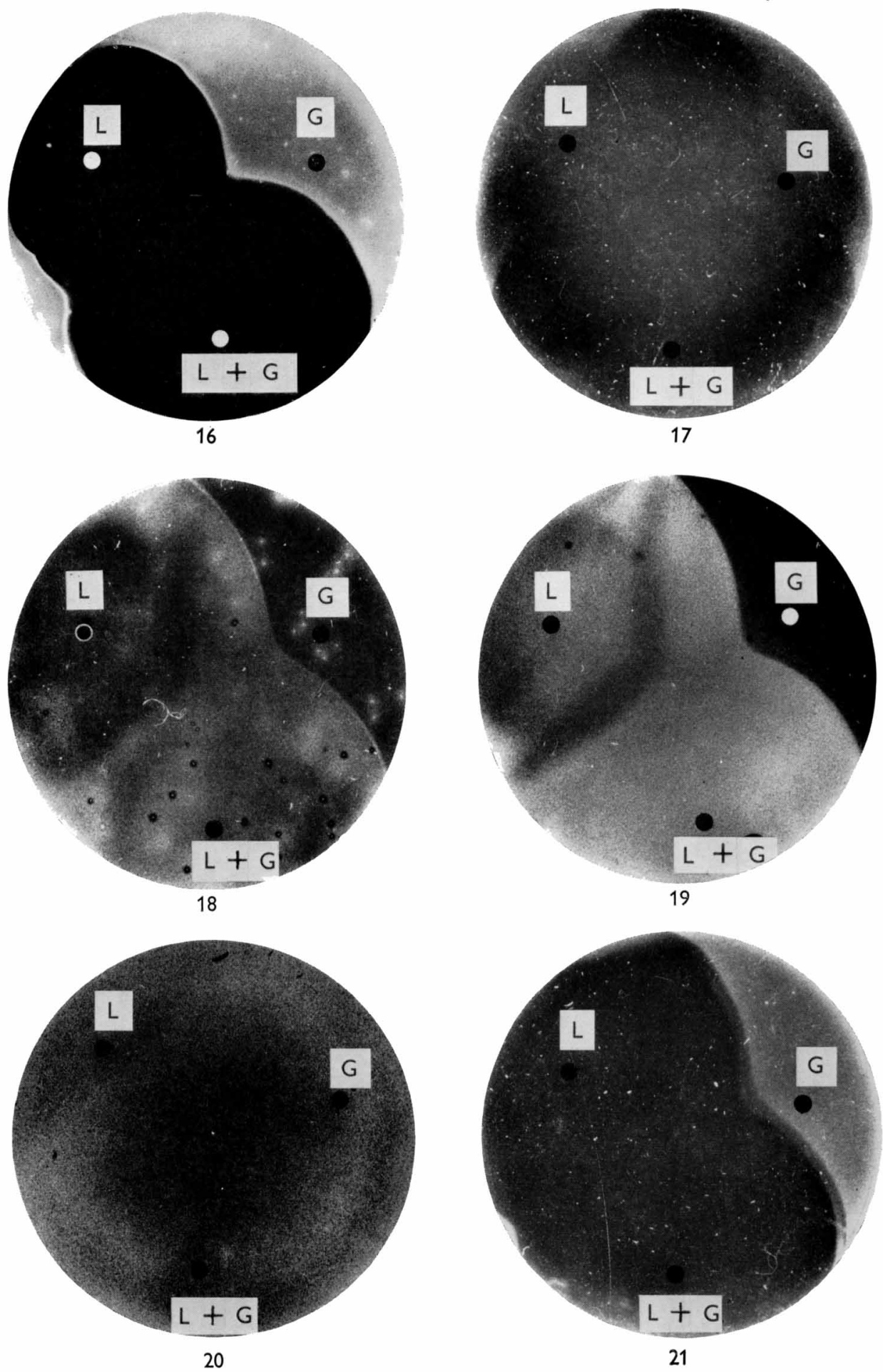

W. B. MOORE 